\title{
Study of the Fermentation Process of Different Fruit Biomasses
}

\author{
Estudo do Processo de Fermentação de Diferentes Biomassas de Frutas
}

\author{
Jailson Marques da Silva ${ }^{\dagger,}$, Nayana de Figueiredo Pereira ${ }^{\dagger}$, David Vilas Boas de Campos ${ }^{\S}$, Antônio \\ Orlando Izolani ", Vinicius Marins Carraro , Carlos Eduardo Cardoso ${ }^{\star}$, Cristiane de Souza Siqueira \\ Pereira $^{\dagger * *}$
}

Como citar esse artigo. Da Silva, JM; Pereira, NF; De Campos, DVB; Izolani, AO; Carraro, VM; Cardoso, CE; Pereira, CSS. Study of the fermentation process of different fruit biomasses. Revista Teccen. 2018 Jul./Dez.; 11 (2): 58-64.

\begin{abstract}
Resumo
Os resíduos orgânicos representam metade dos resíduos urbanos gerados no Brasil, sendo 30\% originados de frutas. Em busca de novas alternativas de reaproveitamento desta biomassa, o presente trabalho avaliou a produção de etanol a partir da biomassa de banana, maçã, laranja e mamão gerado em estabelecimentos comerciais da cidade de Vassouras/RJ. Os produtos destilados oriundos do processo de fermentação da biomassa de frutas foram caracterizados com relação aos compostos voláteis (acetaldeído, acetona, acetato de etila, etanol, metanol, álcoois superiores isopropanol e isoamílico). Os resultados apresentaram um teor álcool promissor de acordo com os trabalhos encontrados na literatura. Palavras-Chave: resíduos, reaproveitamento, frutas, química verde
\end{abstract}

\begin{abstract}
Organic waste accounts for half of the urban waste generated in Brazil, 30\% of which originates from fruit. In the search for new alternatives to reuse this biomass, the present study evaluated ethanol production from banana, apple, orange and papaya biomasses generated in commercial establishments in the city of Vassouras/RJ. The distilled products from the fruit biomass fermentation process were characterized regarding volatile compounds, acetaldehyde, acetone, ethyl acetate, ethanol, methanol, higher alcohols, isopropanol and isoamylic. The results indicate promising alcohol content according to the literature..

Keywords: waste, reuse, bioethanol, fruit, green chemical.
\end{abstract}

\section{Introduction}

Organic waste has become a recent and severe problem, due to population increases leading to increased food consumption and, consequently, increased waste. One of the most important problems faced by society in this regard is the correct destination for this waste. One way to prevent this biomass from being disposed of improperly would be to transform them in energy sources (Schramm, 1992).

Waste and the inappropriate disposal of energy sources have led to a reevaluation of the waste culture, with the emergence of innovative technologies in this area, aiming at waste reuse, leading to novel renewable energy sources. Concerns regarding environmental issues and increased oil costs justify the interest in obtaining fuels from renewable sources. In this context, ethanol produced from biomass appears as a promising alternative to replace oil products, thus contributing to reducing pollutant emissions.

According to the United Nations Food and Agriculture Organization (FAO), Brazil loses around $64 \%$ of its entire annual food production. Data indicate that vegetable losses account for an average of $37 \mathrm{~kg} /$ hab/year, while the consumption of these foods does not exceed $35 \mathrm{~kg}$ during the same timeframe (FAO, 2008). The integral use of fruits and vegetables (pulp, husks, stems and leaves) for the development of new products is a clean technological alternative within reach of several segments, both industrial and residential (Silva, 2009).

Due to climate, Brazil is noteworthy as a major fruit producer. However, most of this production is lost, either by natural degradation, pulping during handling, or imperfections that make commercialization unfeasible. The consumer market is very demanding as to fruit quality, and products undergo a careful selection based on disease infestations and physical defects, which has led to a $30 \%$ discard of the national production in the

Afiliação dos autores: † Universidade de Vassouras, Curso de Engenharia Química, Vassouras - RJ, Brasil

¥ Curso de Mestrado Profissional em Ciências Ambientais, Universidade de Vassouras - RJ, Brasil;

$\S$ EMBRAPA SOLOS - Rio de Janeiro, Brasil. 
last years. Among fruits that suffer the most wastes are bananas, oranges, apples and papayas.

During alcoholic fermentation, yeast produce secondary products, such as glycerol, high alcohols (whose mixture forms fusel oil) and organic acids, with an emphasis on succinic and pyruvic acid. These compounds can be marketed to the chemistry industry, especially fusel oil (consisting predominantly of isoamyl alcohol), which can be used to produce solvents, varnishes and perfume fixatives (Gutierrez, 1991; Usina São Luiz, 2014).

In this context, this study proposes the recovery and use of discarded biomass from a commercial establishment in the city of Vassouras/RJ for bioethanol production through alcoholic sugar fermentation by the yeast Saccharomyces cerevisiae.

\section{Materials and Methods}

This study was carried out in the physicochemistry laboratory at the Vassouras University, where the fermentation and distillation tests were conducted.

As a preliminary test, the process fermentation of main Brazilian waste fruits (banana, papaya, apple and orange) was evaluated aiming to investigate which fruit or mixture of fruits results in greater alcohol and fermentation byproduct yields.

Improper fruits for commercialization used herein were kindly provided by a fruit establishment located in the city of Vassouras - RJ. The establishment in question discards around $50 \mathrm{~kg}$ of solid waste per week, without reuse. A total of eight samples were prepared using different combinations of the four fruits and samples containing $100 \%$ of each fruit, resulting in 8 biomasses 300 grams each. The samples were classified displayed in Table 1.

The samples were ground with the addition of $100 \mathrm{~mL}$ distilled water. Figure 1 shows the fruits and the worts prepared for the fermentation process.

One hundred grams of biological yeast containing S. cerevisiae and $400 \mathrm{~mL}$ of distillate water were added to each wort sample. The samples were then sealed with film paper and stored at room temperature for 7 days. After fermentation, the filtered wort was distilled for 4 h.

The effects of fermentation time and inocula mass for ethanol and distillation product production were statistically evaluated through an experimental design. The $\mathrm{pH}$ and the ${ }^{\circ} \mathrm{Brix}$ during fermentation were analyzed. The ethanol content of the distilled samples was determined through density and then calculated the specific mass. The results are found through the AOAC reference table, where the value obtained in the specific mass calculation is used as a parameter, in its respective column, indicating its alcohol content in a 20/20 column (Table AOAC Official Methods of Analysis- Reference Tables- Appendix C- Ethanol AOAC $\left.60^{\circ} \mathrm{F}(\% \mathrm{v} / \mathrm{v})\right)$.

Volatile compounds and alcohols from the alcoholic fermentation were analyzed on a Agilent 7890B chromatograph. The standard solution concentrations were as follows: acetaldehyde: $0.10149 \mathrm{mg} / \mathrm{L}$, acetone: $0.2011195 \mathrm{mg} / \mathrm{L}$, ethyl acetate: $0.1012986 \mathrm{mg} / \mathrm{L}$, methanol: $0.2003198 \mathrm{mg} / \mathrm{L}$, propanol: $0.201798 \mathrm{mg} / \mathrm{L}$, isobutanol: $0.1016982 \mathrm{mg} / \mathrm{L}$, isoamyl acetate: 0.1014984 $\mathrm{mg} / \mathrm{L}$ and isoamyl alcohol: $0.09996 \mathrm{mg} / \mathrm{L}$. The capillary column was set at a maximum flow of $2 \mathrm{~mL} / \mathrm{min}$, with the following operating conditions established through previously performed tests: initial temperature of $60^{\circ} \mathrm{C}$ during the first 10 minutes until the first ramp of $70{ }^{\circ} \mathrm{C}$, for 3 minutes, with a second ramp until $80^{\circ} \mathrm{C}$, maintained

Table 1. Fruits and their respective wortsprepared for fermentation.

\begin{tabular}{cc}
\hline Sample & Composition \\
\hline A1 & $100 \%$ banana \\
A2 & $100 \%$ apple \\
A3 & $100 \%$ papaya \\
A4 & $100 \%$ orange \\
A5 & $55 \%$ banana, $15 \%$ apple, $15 \%$ papaya, $15 \%$ orange \\
A6 & $55 \%$ apple, $15 \%$ banana, $15 \%$ papaya, $15 \%$ orange \\
A7 & $55 \%$ papaya, $15 \%$ banana, $15 \%$ apple, $15 \%$ orange \\
A8 & $55 \%$ orange, $15 \%$ banana, $15 \%$ apple, $15 \%$ papaya \\
\hline
\end{tabular}


Figure 1. Fruits and their respective worts prepared for fermentation

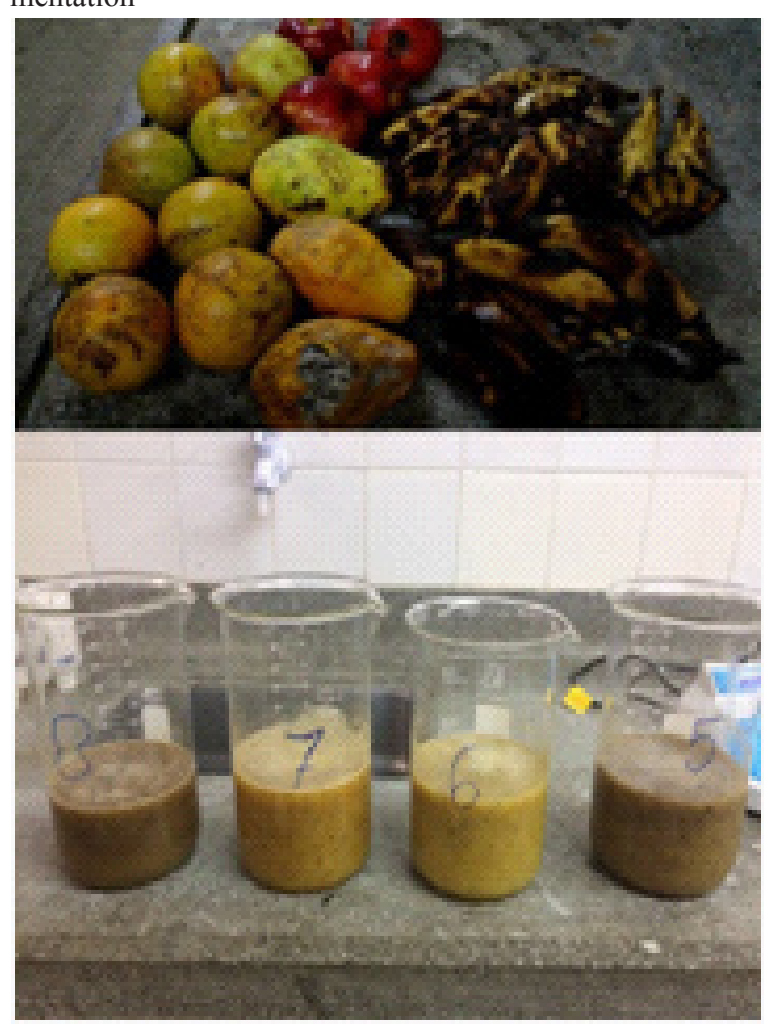

Table 2. Results for $\mathrm{pH},{ }^{\circ}$ Brix and alcohol produced through the fermentation and distillation processes in the fruit waste samples evaluated herein.

\begin{tabular}{cccc}
\hline Samples & Initial $\mathbf{~ H ~}$ & ${ }^{\circ}$ Brix & Final pH \\
\hline A1 & 4.85 & 10.32 & 4.18 \\
A2 & 3.81 & 5.32 & 3.03 \\
A3 & 5.08 & 4.02 & 3.84 \\
A4 & 3.77 & 4.52 & 3.25 \\
A5 & 4.58 & 5.84 & 4.79 \\
A6 & 4.21 & 5.85 & 4.05 \\
A7 & 4.61 & 4.48 & 4.92 \\
A8 & 3.87 & 5.56 & 4.33 \\
\hline
\end{tabular}

for 5 minutes and finally a last ramp up to $120^{\circ} \mathrm{C}$ for 10 minutes. The set rate for ramp temperature was of $10^{\circ} \mathrm{C} / \mathrm{min}$. The FID detector point is given at 100 ${ }^{\circ} \mathrm{C}$. The GC cycle from the headspace to the final race time was of 34.33 minutes. Nitrogen was used as the entrainment gas.

\section{Results and Discussion}

Concerning $\mathrm{pH}$, the results obtained herein corroborate literature data as quoted by Torres et al.
(2006). According to these authors, fermented fruit usually presents $\mathrm{pH}$ ranging between 3.0 and 4.0. However, variations in alcohol content reported by different studies are observed, such as the study by Azevedo (2007), who obtained 5\% alcohol from persimmon biomass, and the report by Bortolini, Sant'anna and Torres (2001), who obtained alcoholic percentages between 38,65 and $47.23 \%$ from kiwifruit. These variations can be explained due to the different operational conditions applied to the fermentation processes. Ethanol $\mathrm{pH}$ and 
concentration after fermentation are in accordance with literature data for fermented fruit. Regarding the remaining ${ }^{\circ}$ Brix, total sugar consumption by yeast was observed.

Debaji and Vimalendra (2011) produced bioethanol from apple and banana residues, with 38\% alcohol yields, while Pezani and Biti (2010) produced bioethanol from tropical fruits at $10 \%$ yields. Misha et al (2012) produced alcohol from pineapple residues $(1.78 \%)$, lemon residues $(1.46 \%)$, and banana peels (3.98\%), while Cruz (2014) obtained satisfactory alcohol percentages from banana, apple, orange, mexican papaya and avocado samples, ranging from 23 to $34 \%$.

The gas chromatography results are exhibited in Table 3. Each result is displayed as a chromatogram (Figures 2 to 9).

According to the ${ }^{\circ}$ Brix analyses, sample A1, comprising $100 \%$ banana, presented higher sugar concentrations and, consequently, higher ethanol volume. Because of this, experiments were carried out to evaluate banana biomass fermentation as a function of the fermentation time and inocula mass.

Variations between the acetaldehyde results can be explained due to its formation process during fermentation. According to Valsechi (1990), this compound is originated from the action of oxidizing enzymes from the yeast itself or from alcohol oxidation by the influence of air oxygen. Therefore, differences may be due to yeast enzyme performance and the influence of external media. Acetaldehyde is consumed throughout the process and simultaneously to its decrease, high alcohols are formed, which in this case comprise propanol, isobutanol and isoamyl alcohol.

\section{Conclusions}

The feasibility of obtaining alcohols from the different combinations of discarded fruit biomasses

Table 3. Chromatograms of the distilled samples evaluated herein.

\begin{tabular}{ccccccccc}
\hline Samples & Acetaldehyde & Acetone & Methanol & $\begin{array}{c}\text { Ethyl } \\
\text { Acetate }\end{array}$ & Propanol & Isobutanol & $\begin{array}{c}\text { Isoamyl } \\
\text { Acetate }\end{array}$ & $\begin{array}{c}\text { Isoamyl } \\
\text { Alcohol }\end{array}$ \\
\hline A1 & 20.029 & 0.047 & 101.867 & 10.202 & 134.623 & 221.379 & 0.027 & 564.104 \\
A2 & 5.726 & 0.009 & 39.712 & 2.841 & 60.495 & 159.850 & 0.016 & 387.197 \\
A3 & 4.732 & - & 54.912 & 1.388 & 105.200 & 280.261 & 0.016 & 382.246 \\
A4 & 12.611 & 0.047 & 116.726 & 2.248 & 95.541 & 231.183 & 0.018 & 454.445 \\
A5 & 26.140 & 0.023 & 59.367 & 36.068 & 60.142 & 240.532 & 0.069 & 426.259 \\
A6 & 25.311 & - & 351.531 & 8.585 & 218.632 & 223.016 & 0.021 & 548.998 \\
A7 & 7.733 & 0.006 & 163.043 & 20.199 & 40.675 & 180.974 & 0.052 & 168.586 \\
A8 & 14.118 & 0.552 & 89.330 & 53.675 & 103.749 & 322.596 & 0.009 & 122.355 \\
\hline
\end{tabular}

Note: the acetone for samples A3 e A6 were not detected.

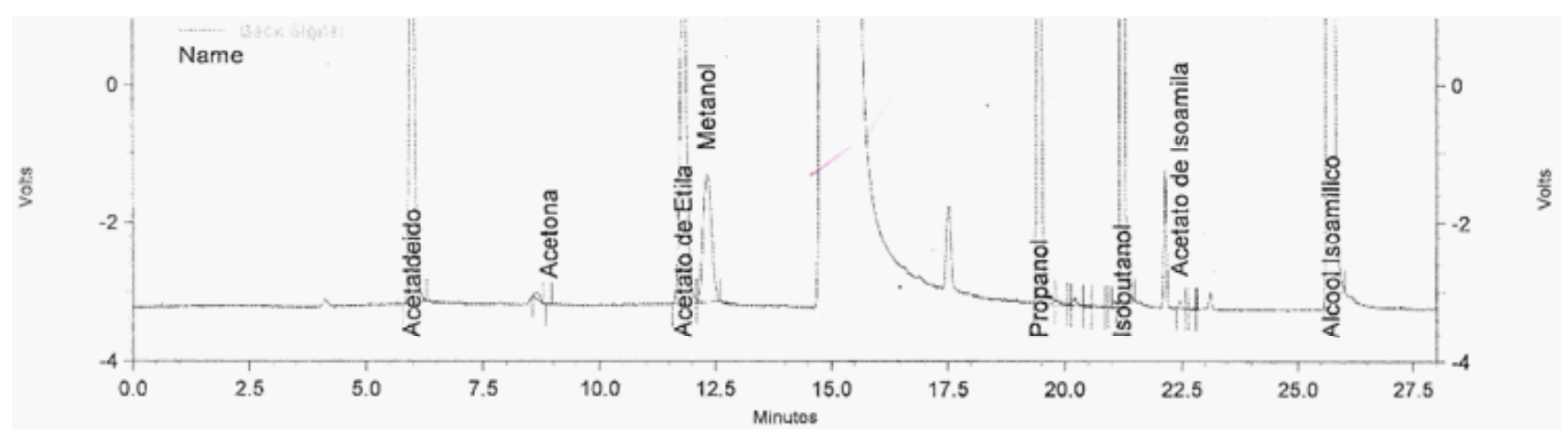

Figure 2. Sample A1 chromatogram. 


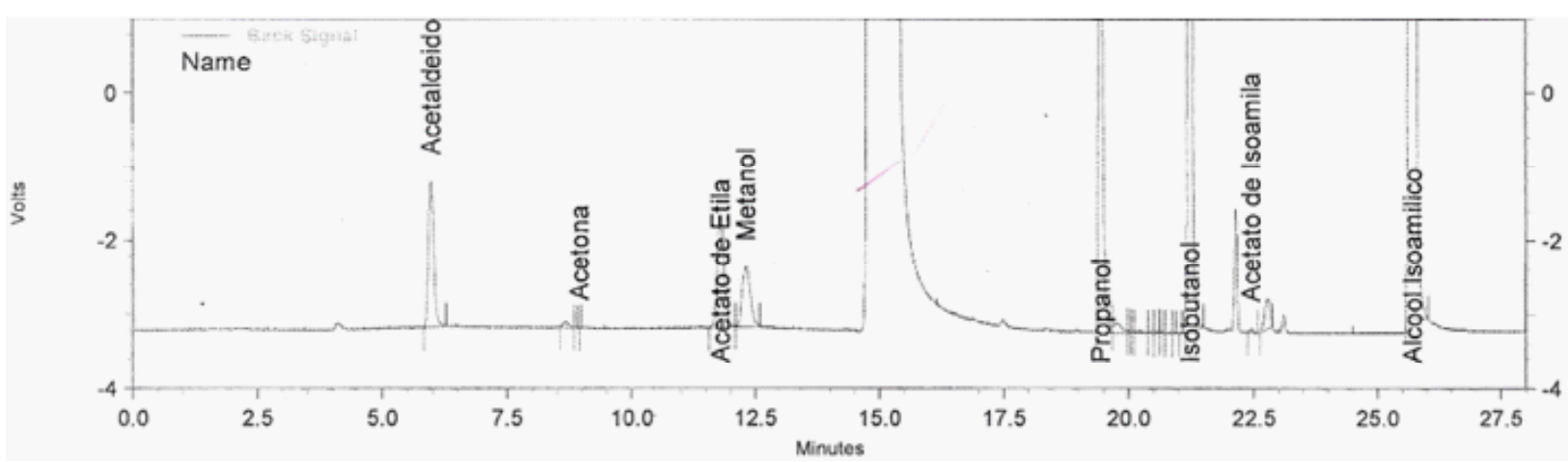

Figure 3. Sample A2 chromatogram.

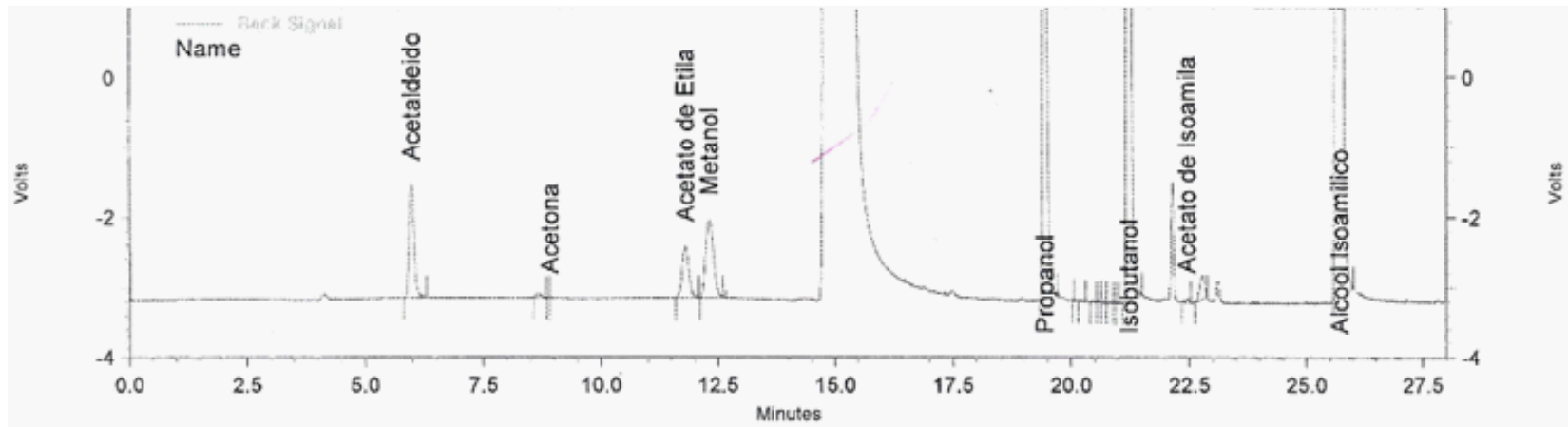

Figure 4. Sample A3 chromatogram.

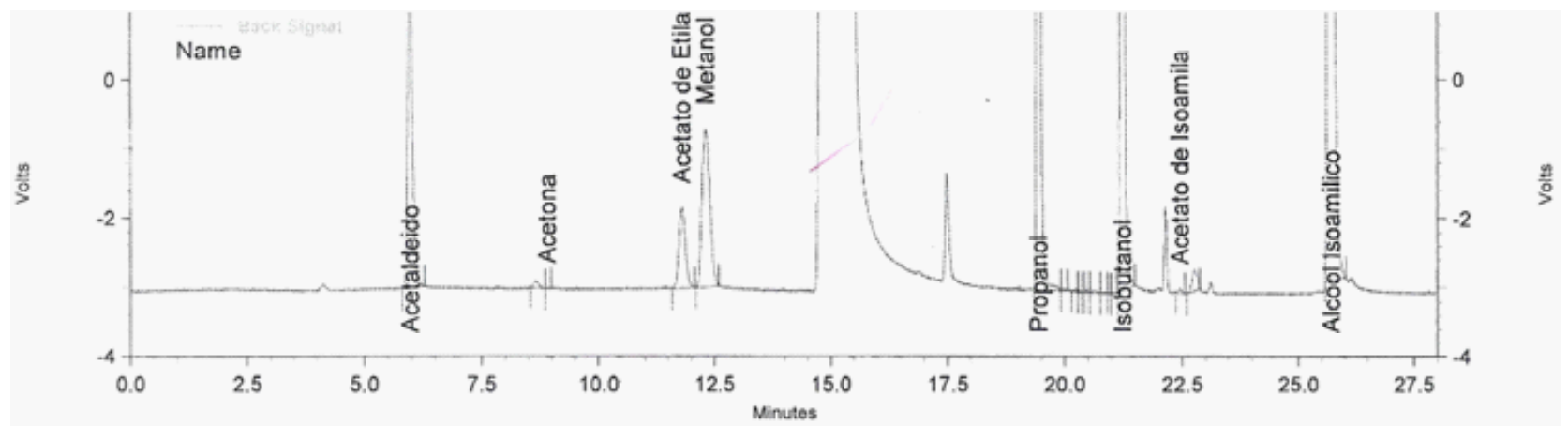

Figure 5. Sample A4 chromatogram.

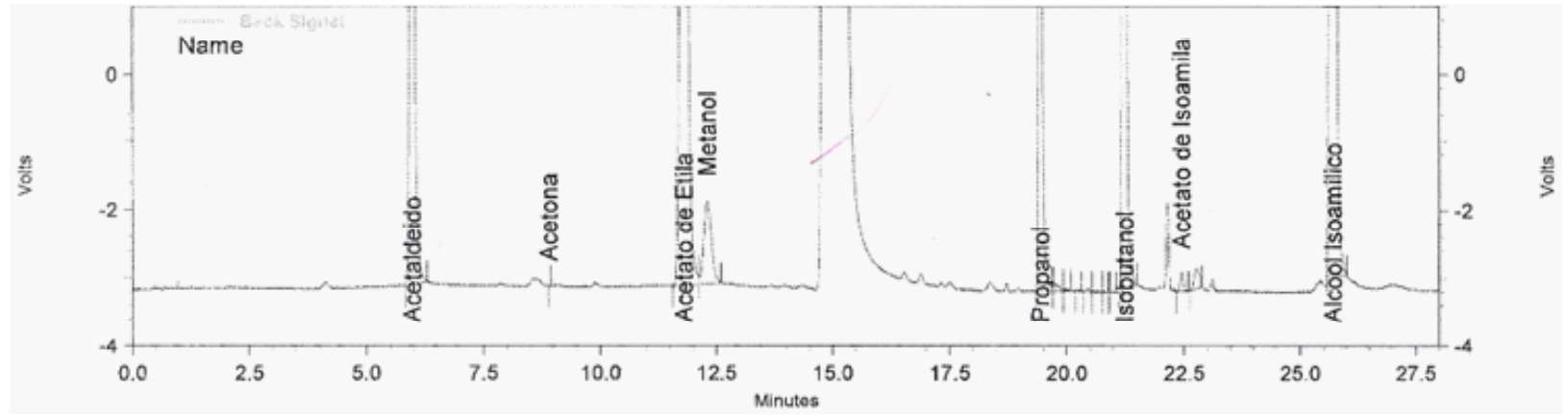

Figure 6. Sample A5 chromatogram. 


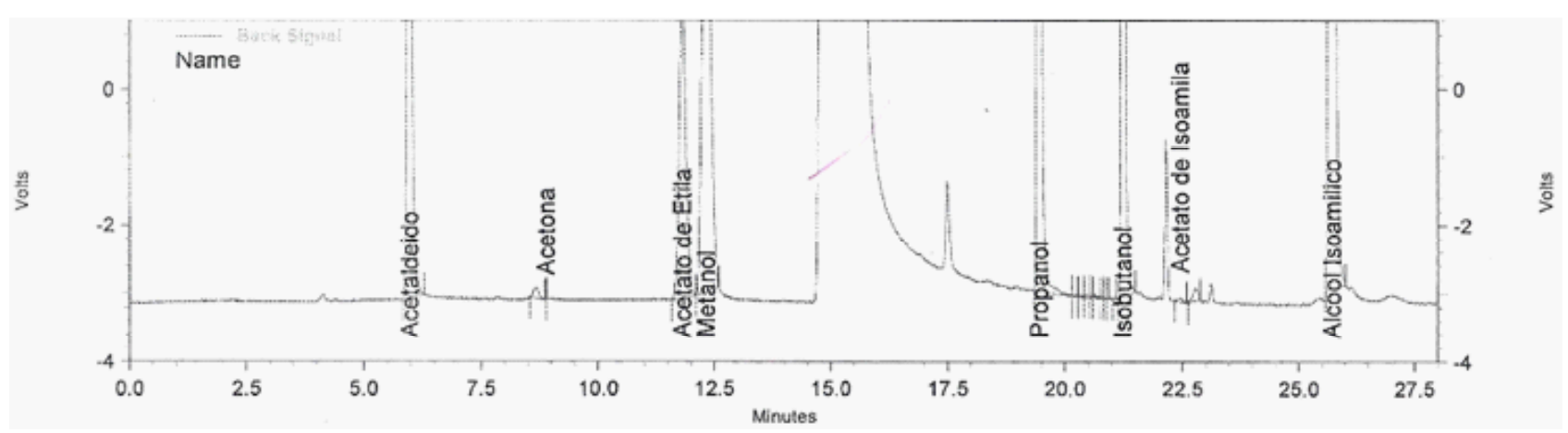

Figure 7. Sample A6 chromatogram.

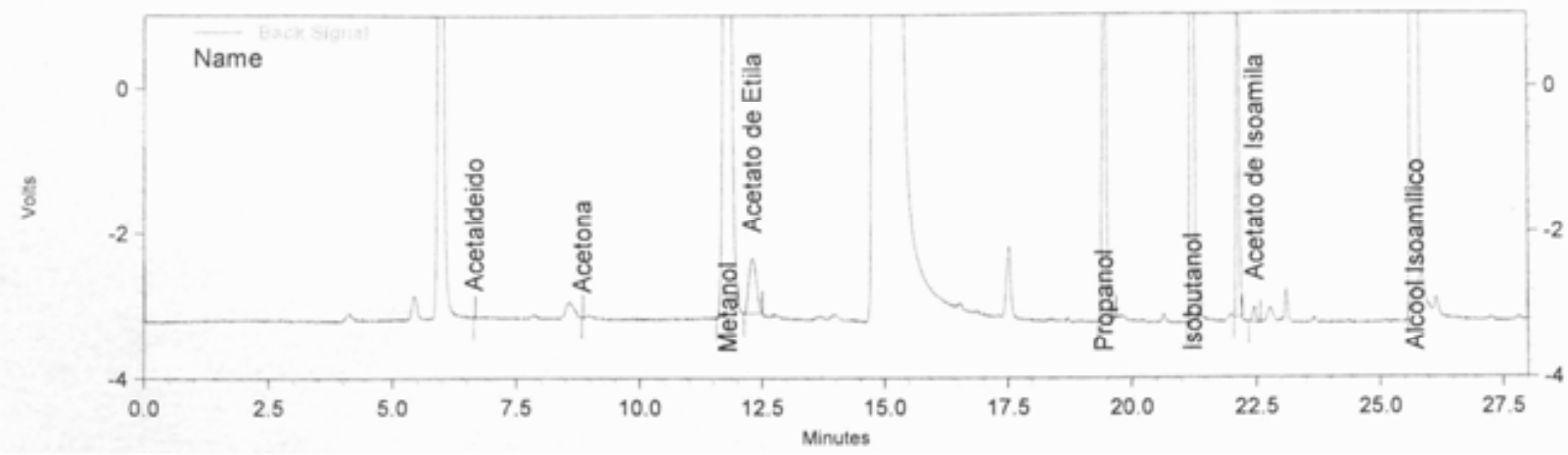

Figure 8. Sample A7 chromatogram.

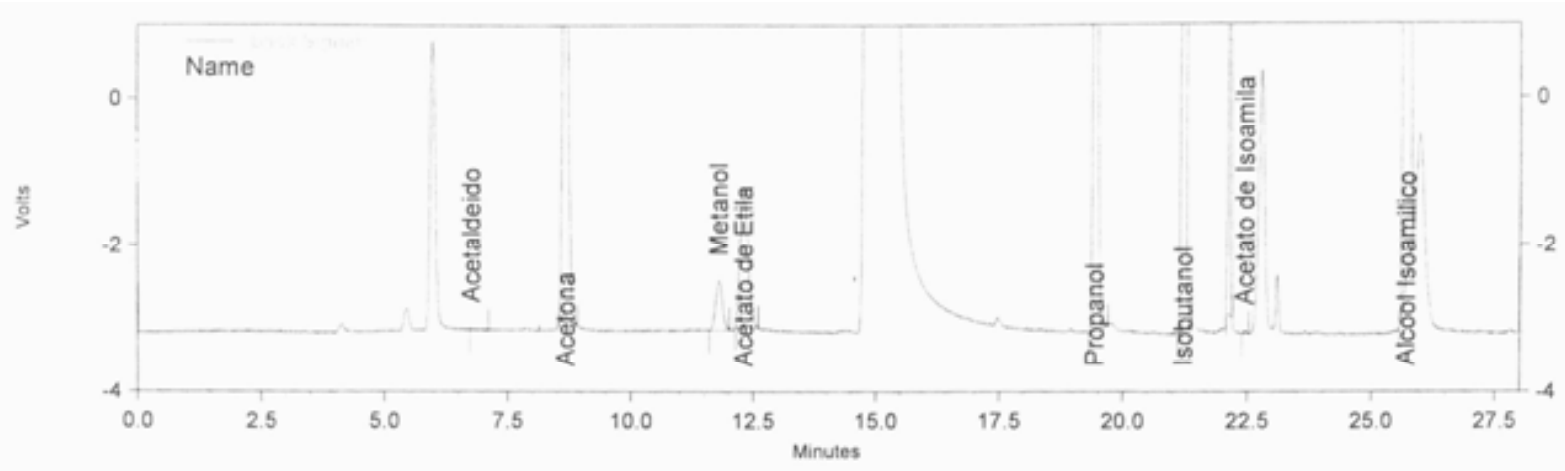

Figure 9. Sample A8 chromatogram.

from an establishment in the city of Vassouras (RJ) was verified. The results presented a promising volume of alcohol, according to studies published in the literature. Biomass reuse for alcohol production contributes as an environmentally correct alternative to add value to these wastes. According to this study we conclude that the banana biomass has the best results, because of its higher sugar concentrations. The analyses indicated the presence of acetaldehyde, acetone, methanol, ethyl acetate, propanol, isobutanol, and isoamyl acetate and isoamyl alcohol, in different concentrations.

\section{References}

ABRELPE - Associação brasileira de empresas pública e resíduos especiais Panorama de resíduos sólidos no Brasil. São Paulo: Abrelpe, 2014.
ABECITRUS (Associação brasileira dos exportadores de cítricos). Frutas e ração animal, 2014.

AGUIAR, A.; FERRAZ, A. Mecanismos envolvidos na biodegradação de materiais lignocelulósicos e aplicações tecnológicas correlatas. Química nova, vol. $34, \mathrm{n}^{\circ} 10$. p. $1729-1738,2011$.

BALAT, M. Production of bioethanol from lignocellulosic materials via the biochemical pathway: A review. Energy conversion and management, $\mathrm{n}^{\circ} 52$. p. $858-875,2011$

BNDES. Bioetanol de cana-de-açúcar: energia para o desenvolvimento sustentável. $1^{\text {a }}$ ed. Rio de Janeiro, 2008.

BRASIL. Constituição (1988). Constituição da república federativa do Brasil. Brasília, DF: Senado, 1988. Disponível em < http://www.planalto.gov. br/ccivil 03/constituicao/Constituicao.htm >

CANTIllanO, R. F. F.; CAMElatTO, D.; MEDEIROS, A. R. M de; PAIVA, E. Efeito do grau de maturação na conservação de maçãs cv. Golden delicious, In: Congresso brasileiro de fruticultura 6. Recife, 1981. Anais. Recife, Sociedade brasileira de fruticultura, p. 845 - 6. 1981. 
CHEFFTEL, J. C; CHEFFTEL, H.; BESANCON, N. P. Introduccion a la bioquimica y tecnologia de los alimentos. Zaragoza: Acríbia, vol. 1. 1977. 333 p. COM - European Commission. Towards a circular economy: a zero waste programme for Europe. Com (2014) 398 final. Bruxelas: com, 2014.

CRUZ, D. Trabalho de conclusão de curso apresentado ao curso de química industrial da Universidade Severino Sombra, para obtenção do grau de química industrial - 2014

DEBAJIT, B. \& VIMALENDRA, M. Production of bio fuel from fruit waste. International journal of advanced biotechnology research, 1, 71 - 74, 2011.

DIAS, M. O. S. et al. Integrated versus stand-alone second generation ethanol production from sugarcane bagasse and trash. Bioresource technology, $\mathrm{n}^{\circ}$ 103. p. $152-161,2012$.

EVARISTO, J. J. Avaliação de diferentes reagentes na extração da pectina do albedo da laranja. Trabalho de conclusão de curso - TCC (Bacharelado em química) - Centro de ciências exatas e naturais do departamento de química. Universidade Regional de Blumenau, 2009.

FRANCELLI, M. Cultivo da banana para o estado do Amazonas - Importância econômica. Revista eletrônica Embrapa mandioca e fruticultura. Sistema de produção, 6. Versão eletrônica 2003

FALLER, A. L. K.; FIALHO, E. Disponibilidade de polifenóis em frutas e hortaliças consumidas no Brasil. Revista saúde pública, São Paulo, vol. 43, $\mathrm{n}^{\mathrm{o}} 2$, p. $211-218,2009$

Ferreira, J. Produção de levana e bioetanol utilizando cascas de banana por Zimomona mobilis. Universidade Estadual Paulista Júlio de Mesquita Filho - São José do Rio Preto, 2013.

FAO. The state of food insecurity in the world. Roma, Itália. 2009.

GONCALVES JUNIOR, C. A. et al. Um estudo das deliberações da câmara setorial do açúcar e do álcool, usando análise de correspondência. Revista de economia e sociologia rural, Piracicaba, vol. 47, nº 1, p. $183-210,2009$.

GUTIERREZ, L. E. Produção de glicerol por linhagens de Saccharomyces durante fermentação alcoólica. Anais ESALQ. vol. 48. p. 55 - 69. Piracicaba, São Paulo, 1991.

HADAR, Y. Chapter 2 - Sources for lignocellulosic raw materials for the production of ethanol. 2013

HERNANDEZ, D. I. M. Efeitos da produção de etanol e biodiesel na produção agropecuária do Brasil. 163 f. Dissertação (Mestrado em agronegócios) Curso de pós-graduação em agronomia e medicina veterinária, Universidade Federal de Brasília, DF, 2008

KEFALAS, H. C.; SOUZA, S. A. D.; DENEKA, L. G.: Resíduos orgânicos na zona costeira: a proposta da compostagem. Simpósio brasileiro de oceanografia, Santos. Anais. Oceanografia e políticas públicas. 2011.

LEITSINGER, M. Iowa group drafts plan of state's biotech future. Center for industrial research and service. Disponível em: https://www.ciras.iastate. edu/publications/biowa.asp, 2003.

MACHADO, P. A. L. Direito ambiental brasileiro. $21^{\text {a }}$ ed. São Paulo: Malheiros, 2013.

MENON, V.; RAO, M. Trends in bioconversion of lignocellulose: Biofuels, platform chemicals e biorefinery concept. Progress in energy and combustion science, $\mathrm{n}^{\circ} 38$. p. $522-550,2012$

MEDINA, J. C.; et al. Banana: da cultura ao processamento e comercialização. $2^{a}$ ed. Campinas, ITAL: p. 1 - 131/198 - 264, 1985.

MILANEZ, A. et. al. Logística para o etanol: situação atual e desafios futuros. Sucroenergético. BNDES Setorial 31.p. 49 - 98, 2010.

MISHRA, J., KUMAR, D., SAMANTA, S., \& VISHWAKARMA, M. K. Um estudo comparativo da produção de etanol a partir de vários resíduos agro utilizando Saccharomyces cerevisiae e a Candida albicans. Jornal de Levedura e de Fungos Research, 3 (2), 12 - 17, 2012.

NELSON, D.; Cox, M. M. L., Princípios de bioquímica $5^{\mathrm{a}}$ ed. São Paulo, Savier, 2011

OLIVEIRA, L. M. et al. Estudo comparativo das diferentes tecnologias utilizadas para produção de etanol. Revista eletrônica do curso de geografia $\mathrm{n}^{\circ}$ 19. Universidade Federal de Goiás, 2012.

PAULILLO, L. F. et al. Análise da competitividade das cadeias de agroenergia no Brasil. In: BUAINAIN, A. M.; BATALHA, M. O. (Coord.). Análise da competitividade das cadeias agroindustriais brasileiras. São Carlos, SP: DEP - UFSCAR/ IE -UNICAMP, p. 119, 2006.

PEREIRA, L. B.; SIMIONI, F. J. e CARIO, S. A. F.. Evolução da produção de maçã em Santa Catarina: novas estratégias em busca de maior competitividade, 2008 .

PEZZANI, H; BITTI, T. M. Recuperação e utilização de resíduos sólidos orgânicos provenientes da indústria de processamento de frutas na produção de etanol. Enciclopédia biosfera, Centro Científico Conhecer - Goiânia, vol. $6, \mathrm{n}^{\mathrm{o}} 10,2010$

RABELO, S. C. Avaliação e otimização de pré-tratamentos e hidrólise enzimática do bagaço de cana-de-açúcar para a produção de etanol de segunda geração. Tese (Doutorado em engenharia química). Universidade Estadual de Campinas. Campinas, São Paulo. 2010

RATHMANN, R et al. Land use competition for production of food and liquid biofuels: An analysis of the arguments in the current debate. Renewable energy, vol. $35, \mathrm{n}^{\circ}$ 1. p. $14-22,2010$.

RIBEIRO, F. DE M.; KRUGLIANSKAS, I. A. Economia circular no contexto europeu: Conceito e potenciais de contribuição na modernização das políticas de resíduos sólidos. Em: XVI Encontro lignocellulose internacional sobre gestão empresarial e meio ambiente (ENGEMA). São Paulo, 2014.

RIBEIRO, H.; BESEN, G. R. Panorama da coleta seletiva no Brasil: Desafios e perspectivas a partir de três estudos de caso. Revista de gestão integrada em saúde do trabalho e meio ambiente. vol. 2, no 4, p. 1 - 18, 2007.

RODRIGUES, J. A. R. Do engenho à biorrefinaria. A usina de açúcar como empreendimento industrial para a geração de produtos bioquímicos e biocombustíveis. Química nova, vol. 34, nº 7. p. 1242 - 1254, 2011.

SCHRAMM, F. R. Ecologia, ética e saúde: O principio da responsabilidade. In: saúde, ambiente e desenvolvimento (M. C. Leal; P. C. Sabroza; R. H. Rodrigues \& P. M. Buss, orgs.), p. 233 - 255, vol. 2, São Paulo: Hucitec/Rio de Janeiro: Abrasco. Disponível: http://www.bvsde.paho.org/bvsacd/cd48/ v11n2a14.pdf, 1992.

SANTANA, N. B. Eficiência da hidrólise de amido de mandioca por diferentes fontes de enzimas e rendimento da fermentação alcoólica para produção de etanol. Dissertação (Magister scientiae). Universidade Federal de Viçosa. Viçosa, Minas Gerais. 2007.

SILVA, M. B. de; RAMOS, A. M. Composição química, textura e aceitação sensorial de doces em massa elaborados com polpa de banana e banana integral. Revista Ceres, Viçosa, vol. 56, nº 5, p. 551 - 554, 2009.

SILVA, N. L. C. Produção de bioetanol de segunda geração a partir de biomassa residual da indústria de celulose. Dissertação (Mestrado em tecnologia de processos químicos e bioquímicos). Universidade Federal do Rio de Janeiro. Rio de Janeiro. 2010.

SOUZA, S. A. M. Mamão no Brasil: distribuição regional da produção e comportamento dos preços no período 1996 - 2005. Informações econômicas, SP, vol. 37, $\mathrm{n}^{\circ}$ 9, 2007.

SOUZA, O.; FEDERIZZI, M.; COELHO, B.; WAGNER, T. M.; WISBECK, E. Biodegradação de resíduos lignocelulósicos gerados na bananicultura e sua valorização para a produção de biogás. Revista brasileira de engenharia agrícola e ambiental, vol. 14, p. 438 - 443, 2010.

TORRES NETO, Alberto B. et al. Cinética e caracterização físico-química do fermentado do pseudofruto do caju (Anacardium occidentale L.). Química nova [online]. vol. 29, n $3,2006$.

VIIKARI, L. et al. Lignocellulosic ethanol: From science to industry. Biomass and Bioenergy, $\mathrm{n}^{\circ}$ 46. p. 13 - 24, 2012.

WALTER, R. H.; RAO, M. A.; SHERMAN, R. M.; COOLEY, H. J. Edible fibers from apple pomace. Journal of food science, vol. 50, p. 747 - 749, 1985 . 